\title{
Uma proposta para o ensino de sistemas lineares sem a teoria de matrizes, via problemas históricos
} A linear system teaching proposal via historical problems and without the use of
matrices theory

\author{
Viviane Simioli Medeiros Campos ${ }^{1}$ e Agamenon Henrique de Carvalho Tavares ${ }^{2}$ \\ 1,2 Universidade Federal do Rio Grande do Norte- RN, Brasil \\ viviane@ccet.ufrn.br; agamenon.tavares@ifrn.edu.br
}

\begin{abstract}
Resumo
Neste artigo, com base no Método chinês apresentado no livro "Os nove capítulos da arte matemática", escrito no século I da era cristã, sugerimos uma sequência de problemas a partir da qual o aluno irá se apropriar dos conceitos matemáticos envolvidos na definição e na resolução de sistemas lineares, sem a necessidade de conhecer a teoria de matrizes e determinantes.
\end{abstract}

Palavras-chave: Método chinês. Sistemas lineares. Operações elementares

\begin{abstract}
In this work, based on the chinese method which was presented in the book that was published in I a.C., titled "the nine chapters", a sequence of problems were suggested in order to introduce definitions and a method of solving linear systems, without introducing neither matrices nor determinants.
\end{abstract}

Keywords: Chinese method. Linear systems. Elementary operations 


\section{Introdução}

O ensino de sistemas lineares no Ensino Médio é feito, por muitos autores, após o estudo de matrizes e de determinantes. Podemos citar como exemplos os livros de Dante (2010), Iezzi (2010) e PAIVA (2010). Dante (2010), para introduzir as operações elementares necessárias para o escalonamento de um sistema linear, usa o fato de algumas dessas operações serem idênticas às permitidas para verificação de umas das propriedades do determinante, apresentadas em seu texto anteriormente. Para esses autores, o contexto histórico é tratado como curiosidade, uma mera ilustração, mas não como metodologia de ensino.

Matrizes e determinantes são conceitos bastante abstratos. Nossa proposta para o Ensino Médio é que sistemas lineares e o método do escalonamento sejam apresentados e validados antes da teoria de matrizes. Seguindo a ordem cronológica, estamos oferecendo ao professor atividades que possibilitem a construção desses conceitos pelo aluno e que despertem nele a percepção da necessidade de uma teoria antes que ela seja sistematizada, fazendo-o entender, ainda que simplificadamente, como o conhecimento matemático se desenvolve.

Nosso estudo tem como ponto de partida a apresentação e a resolução detalhada de quatro problemas através do Método Chinês. O Método Chinês era uma ferramenta matemática utilizada para resolver problemas do dia a dia e foi apresentado no livro "Os nove capítulos da arte matemática" de Liu Hui (Carrera (2009)), publicado na China durante o século I da era cristã e, conforme Eves (2004), "o mais importante dos textos de matemática chinês".

Ao final da apresentação e resolução de cada um dos problemas, fazemos uma nova leitura dos mesmos com o objetivo de introduzir, para os alunos, as noções de incógnitas e equações, ou seja, de sistemas lineares.

Definindo formalmente as manipulações matemáticas utilizadas pelos chineses na resolução dos problemas, chegamos ao que conhecemos hoje como operações elementares e provamos, na seção 43 , que essas operações quando aplicadas às equações de um sistema linear, o transformam em um outro sistema, cuja as soluções são as mesmas do sistema inicial.

Apresentamos assim uma sequência didática para o estudo de sistemas lineares e o método do escalonamento.

\section{O Método Chinês}

Nesta seção, estudamos o método apresentado em "Os nove capítulos da arte matemática"e que era utilizado para a resolução de problemas práticos do dia a dia dos autores. Nosso contato com a obra se deu através de Carrera (2009) e Eves (2004).

Os chineses consideraram somente problemas envolvendo o mesmo número de situações descritas e valores desconhecidos a serem calculados e não indicaram o motivo desses sistemas poderem ser resolvidos.

As soluções dos problemas pelos chineses basearamse em dois métodos: o shinjutsu ou método direto (substituição direta de valores a serem calculados) e o kyojutsu ou método indireto (simplificação das situações descritas). Nesse trabalho, estudamos problemas que foram resolvidos segundo esse último método.

Realizamos o estudo do Método Chinês resolvendo quatro problemas. Os dois primeiros pertencem a obra citada e fizemos simplesmente repetir o passo-a-passo da resolução com o objetivo de entendermos as operações matemáticas executadas.

Os dois últimos problemas foram criados para exercitarmos método de resolução chinês. Durante a resolução de cada um dos quatro problemas reescrevemos seus enunciados usando letras para representarem as quantidades procuradas, é o que chamamos modelagem.

Problema I. Três feixes de uma colheita de boa qualidade, dois feixes de uma de qualidade regular e um feixe de uma de má qualidade são vendidos por 39 dou. Dois feixes de boa, três de regular e um de má qualidade são vendidos por 34 dou. Um feixe de boa, dois de regular e três de má são vendidos por 26 dou. Qual o preço do feixe para cada uma das qualidades? (EVES, 2004, p. 268).

Segundo o método chinês, o problema deve ser representado, como a seguir.

$\begin{array}{ccc}1 & 2 & 3 \\ 2 & 3 & 2 \\ 3 & 1 & 1 \\ 26 & 34 & 39\end{array}$

mas para ser mais didático apresentamos os dados no seguinte formato

$\begin{array}{cccc} & 1^{\mathrm{a}} & 2^{\mathrm{a}} & 3^{\mathrm{a}} \\ \text { Boa Qualidade } & 1 & 2 & 3 \\ \text { Qualidade Regular } & 2 & 3 & 2 \\ \text { Má Qualidade } & 3 & 1 & 1 \\ & 26 & 34 & 39\end{array}$

Montada a tabela, utilizamos os seguintes passos:

$1^{\circ}$ Passo: multiplicamos todos os termos da coluna central $(2,3,1,34)$ pelo primeiro termo da coluna direita (3), obtendo $(6,9,3,102)$.

$\begin{array}{cccc} & 1^{\mathrm{a}} & 2^{\mathrm{a}} & 3^{\mathrm{a}} \\ \text { Boa Qualidade } & 1 & 6 & 3 \\ \text { Qualidade Regular } & 2 & 9 & 2 \\ \text { Má Qualidade } & 3 & 3 & 1 \\ & 26 & 102 & 39\end{array}$


$2^{\circ}$ Passo: subtraímos o número à direita de cada um dos números do centro, obtendo, no centro, $(6-3=$ $3 ; 9-2=7 ; 3-1=2 ; 102-39=63)$.

$\begin{array}{cccc} & 1^{\mathrm{a}} & 2^{\mathrm{a}} & 3^{\mathrm{a}} \\ \text { Boa Qualidade } & 1 & 3 & 3 \\ \text { Qualidade Regular } & 2 & 7 & 2 \\ \text { Má Qualidade } & 3 & 2 & 1 \\ & 26 & 63 & 39\end{array}$

$3^{\circ}$ Passo: repetimos mais uma vez o $2^{\circ}$ passo até que o primeiro número da coluna central seja eliminado $(3-3=0 ; 7-2=5 ; 2-1=1 ; 63-39=24)$.

$\begin{array}{cccc} & 1^{\mathrm{a}} & 2^{\mathrm{a}} & 3^{\mathrm{a}} \\ \text { Boa Qualidade } & 1 & 0 & 3 \\ \text { Qualidade Regular } & 2 & 5 & 2 \\ \text { Má Qualidade } & 3 & 1 & 1 \\ & 26 & 24 & 39\end{array}$

Seguimos repetindo os dois primeiros passos, agora entre a $1^{a}$ e a $3^{a}$ colunas com o objetivo de eliminar o primeiro elemento da $1^{a}$ coluna.

$1^{\mathrm{O}}$ passo: multiplicamos todos os termos da primeira coluna $(1,2,3,26)$ pelo primeiro termo da coluna ä direita (3), obtendo $(3,6,9,78)$.

$\begin{array}{cccc} & 1^{\mathrm{a}} & 2^{\mathrm{a}} & 3^{\mathrm{a}} \\ \text { Boa Qualidade } & 3 & 0 & 3 \\ \text { Qualidade Regular } & 6 & 5 & 2 \\ \text { Má Qualidade } & 9 & 1 & 1 \\ & 78 & 24 & 39\end{array}$

$2^{\circ}$ Passo: subtrair o número à direita de cada um dos números da primeira coluna, obtendo $(3-3=0 ; 6-2=$ $4 ; 9-1=8 ; 78-39=39)$.

$\begin{array}{cccc} & 1^{\mathrm{a}} & 2^{\mathrm{a}} & 3^{\mathrm{a}} \\ \text { Boa Qualidade } & 0 & 0 & 3 \\ \text { Qualidade Regular } & 4 & 5 & 2 \\ \text { Má Qualidade } & 8 & 1 & 1 \\ & 39 & 24 & 39\end{array}$

Finalmente, utilizamos os três passos iniciais, agora entre a $1^{a}$ colunas e a $2^{a}$ coluna, até eliminar o segundo número da $1^{a}$ coluna.

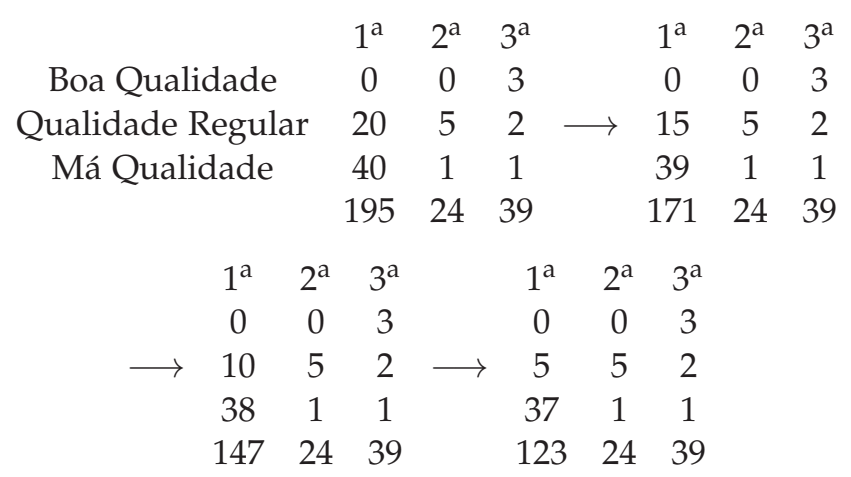

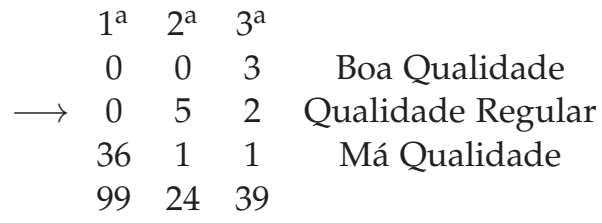

O preço do feixe de má qualidade é $\frac{99}{36}$, que equivale a 2,75 dou.

O valor do feixe de qualidade regular é determinado por substituição:

(Feixe de qualidade regular) $\times 5+2,75=24$

(Feixe de qualidade regular) $\times 5=24-2,75$

(Feixe de qualidade regular) $\times 5=21,25$

(Feixe de qualidade regular) $=\frac{21,25}{5}$

(Feixe de qualidade regular) $=4,25$

Portanto o feixe de qualidade regular custa 4,25 dou.

$\mathrm{O}$ valor do feixe de boa qualidade é determinado por substituição:

(Feixe de boa qualidade $) \times 3+2.4,25+2,75=39$

(Feixe de boa qualidade) $\times 3+8,5+2,75=39$

(Feixe de boa qualidade) $\times 3+11,25=39$

(Feixe de boa qualidade) $\times 3=39-11,25$

(Feixe de boa qualidade) $\times 3=27,75$

(Feixe de boa qualidade $)=\frac{27,75}{3}$

(Feixe de boa qualidade $)=9,25$

Portanto o feixe de boa qualidade custa 9,25 dou.

Para esse problema proposto pelos chineses, é possível atribuir uma letra ao preço indicado para cada feixe com qualidade diferente:

Preço dos feixes de boa qualidade: $x$.

Preço dos feixes de qualidade regular: $y$.

Preço dos feixes de má qualidade: $z$.

Segundo o texto do problema, teríamos uma representação para o problema com as igualdades a seguir.

a) Soma de 3 feixes da colheita boa, 2 feixes da regular e 1 feixe da colheita de má qualidade, totalizando 39 dou: $3 x+2 y+z=39$;

b) Soma de 2 feixes da boa, 3 da regular e 1 da colheita de má qualidade, totalizando 34 dou: $2 x+3 y+$ $z=34$.

c) Soma de 1 feixe da boa, 2 da regular e 3 da colheita de má qualidade, totalizando 26 dou: $x+2 y+3 z=26$.

Assim, o problema pode ser expresso da seguinte forma.

$$
\left\{\begin{aligned}
3 x+2 y+z & =39 \\
2 x+3 y+z & =34 \\
x+2 y+3 z & =26
\end{aligned}\right.
$$

Verifiquemos se os valores encontrados realmente satisfazem às equações.

Preço dos feixes de boa qualidade: 9,25 dou.

Preço dos feixes de qualidade regular: 4,25 dou.

Preço dos feixes de má qualidade: 2,75 dou. 
a) Soma de 3 feixes da colheita boa, 2 feixes da de qualidade regular e 1 feixe da colheita de má qualidade totalizando 39 dou:

$$
3 \times 9,25+2 \times 4,25+2,75=27,75+8,50+2,75=39 ;
$$

b) Soma de 2 feixes da boa, 3 da regular e 1 da colheita de má qualidade totalizando 34 dou:

$$
2 \times 9,25+3 \times 4,25+2,75=18,50+12,75+2,75=34 .
$$

c) Soma de 1 feixe da boa, 2 da regular e 3 da colheita de má qualidade totalizando 26 dou:

$$
9,25+2 \times 4,25+3 \times 2,75=9,25+8,50+8,25=26 .
$$

Vamos acompanhar como os chineses resolveram mais um problema.

Problema II. Suponha que cinco ovelhas, quatro patos, três galinhas e dois coelhos valem 1496 moedas; quatro ovelhas, dois patos, seis galinhas e três coelhos valem 1175; três ovelhas, um pato, sete galinhas e cinco coelhos valem 958; duas ovelhas, três patos, cinco galinhas e um coelho valem 861. Então, qual é o preço de uma ovelha, um pato, uma galinha e um coelho? (CARRERA, 2009, p. 181).

Segundo o Método Chinês e usando o nosso formato, o problema é representado por

$\begin{array}{ccccc} & 1^{\mathrm{a}} & 2^{\mathrm{a}} & 3^{\mathrm{a}} & 4^{\mathrm{a}} \\ \text { Ovelhas } & 2 & 3 & 4 & 5 \\ \text { Patos } & 3 & 1 & 2 & 4 \\ \text { Galinhas } & 5 & 7 & 6 & 3 \\ \text { Coelhos } & 1 & 5 & 3 & 2 \\ & 861 & 958 & 1175 & 1496\end{array}$

Montada essa tabela, utilizamos os mesmos passos que seguimos no problema anterior, realizando as multiplicações e subtrações (ou somas) exigidas.

$1^{\mathrm{o}}$ Passo: multiplicamos todos os termos da $3^{\mathrm{a}}$ coluna $(4,2,6,3,1175)$ pelo primeiro termo da coluna à direita (5), obtendo-se $(20,10,30,15,5875)$.

$\begin{array}{ccccc} & 1^{\mathrm{a}} & 2^{\mathrm{a}} & 3^{\mathrm{a}} & 4^{\mathrm{a}} \\ \text { Ovelhas } & 2 & 3 & 20 & 5 \\ \text { Patos } & 3 & 1 & 10 & 4 \\ \text { Galinhas } & 5 & 7 & 30 & 3 \\ \text { Coelhos } & 1 & 5 & 15 & 2 \\ & 861 & 958 & 5875 & 1496\end{array}$

$2^{\circ}$ Passo: subtraímos o número à direita de cada um dos números da $3^{a}$ coluna, obtendo, em seus lugares, respectivamente, $(20-5=15 ; 10-4=6 ; 30-3=$ $27 ; 15-2=13 ; 5875-1496=4379)$.

$\begin{array}{ccccc} & 1^{\mathrm{a}} & 2^{\mathrm{a}} & 3^{\mathrm{a}} & 4^{\mathrm{a}} \\ \text { Ovelhas } & 2 & 3 & 15 & 5 \\ \text { Patos } & 3 & 1 & 6 & 4 \\ \text { Galinhas } & 5 & 7 & 27 & 3 \\ \text { Coelhos } & 1 & 5 & 13 & 2 \\ & 861 & 958 & 4379 & 1496\end{array}$

$3^{\circ}$ Passo: repetimos continuamente o $2^{\mathrm{O}}$ passo até que o primeiro número da $3^{\mathrm{a}}$ coluna seja eliminado.

$(15-5=10 ; 6-4=2 ; 27-3=24 ; 13-2=11 ; 4379-$ $1496=2883)$.

$\begin{array}{ccccc} & 1^{\mathrm{a}} & 2^{\mathrm{a}} & 3^{\mathrm{a}} & 4^{\mathrm{a}} \\ \text { Ovelhas } & 2 & 3 & 10 & 5 \\ \text { Patos } & 3 & 1 & 2 & 4 \\ \text { Galinhas } & 5 & 7 & 24 & 3 \\ \text { Coelhos } & 1 & 5 & 11 & 2 \\ & 861 & 958 & 2883 & 1496\end{array}$

$$
\begin{aligned}
& (10-5=5 ; 2-4=-2 ; 24-3=21 ; 11-2= \\
& 9 ; 2883-1496=1387) .
\end{aligned}
$$

$\begin{array}{ccccc} & 1^{\mathrm{a}} & 2^{\mathrm{a}} & 3^{\mathrm{a}} & 4^{\mathrm{a}} \\ \text { Ovelhas } & 2 & 3 & 5 & 5 \\ \text { Patos } & 3 & 1 & -2 & 4 \\ \text { Galinhas } & 5 & 7 & 21 & 3 \\ \text { Coelhos } & 1 & 5 & 9 & 2 \\ & 861 & 958 & 1387 & 1496\end{array}$

$$
\begin{aligned}
& (5-5=0 ;-2-4=-6 ; 21-3=18 ; 9-2=7 ; 1387- \\
& 1496=-109) .
\end{aligned}
$$

\begin{tabular}{|c|c|c|c|}
\hline $1^{\mathrm{a}}$ & $2^{\mathrm{a}}$ & $3^{a}$ & $4^{\mathrm{a}}$ \\
\hline 2 & 10 & 0 & 5 \\
\hline 3 & 1 & -6 & 4 \\
\hline 5 & 32 & 18 & 3 \\
\hline 1 & 23 & 7 & 2 \\
\hline 861 & 3294 & -109 & 1496 \\
\hline
\end{tabular}

$\begin{array}{ccccc} & 1^{\mathrm{a}} & 2^{\mathrm{a}} & 3^{\mathrm{a}} & 4^{\mathrm{a}} \\ \text { Ovelhas } & 2 & 3 & 0 & 5 \\ \text { Patos } & 3 & 1 & -6 & 4 \\ \text { Galinhas } & 5 & 7 & 18 & 3 \\ \text { Coelhos } & 1 & 5 & 7 & 2 \\ & 861 & 958 & -109 & 1496\end{array}$

Seguimos repetindo os dois primeiros passos, agora entre a $2^{a}$ e a $4^{a}$ colunas, eliminando-se o primeiro elemento da $2^{a}$ coluna.

$\begin{array}{cccccc} & 1^{\mathrm{a}} & 2^{\mathrm{a}} & 3^{\mathrm{a}} & 4^{\mathrm{a}} & \\ \text { Ovelhas } & 2 & 15 & 0 & 5 & \\ \text { Patos } & 3 & 5 & -6 & 4 & \\ \text { Galinhas } & 5 & 35 & 18 & 3 \\ \text { Coelhos } & 1 & 25 & 7 & 2 & \\ & 861 & 4790 & -109 & 1496 & \end{array}$




$$
\begin{aligned}
& 1^{\mathrm{a}} \quad 2^{\mathrm{a}} \quad 3^{\mathrm{a}} \quad 4^{\mathrm{a}} \\
& 250 \quad 5 \\
& \longrightarrow \begin{array}{ccccc}
3 & -3 & -6 & 4 \\
5 & 29 & 18 & 3
\end{array} \longrightarrow \\
& \begin{array}{lllll}
1 & 21 & 7 & 2
\end{array} \\
& \begin{array}{llll}
861 & 1798 & -109 & 1496
\end{array}
\end{aligned}
$$

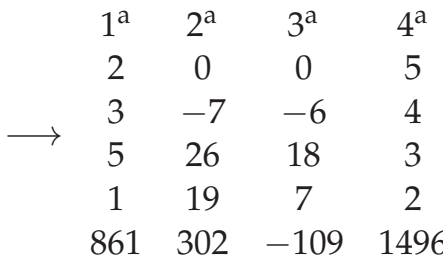

Seguimos repetindo os dois primeiros passos, agora entre a $1^{a}$ e a $4^{a}$ colunas, eliminando o primeiro elemento da $1^{a}$ coluna.

$$
\begin{array}{ccccc} 
& 1^{\mathrm{a}} & 2^{\mathrm{a}} & 3^{\mathrm{a}} & 4^{\mathrm{a}} \\
\text { Ovelhas } & 10 & 0 & 0 & 5 \\
\text { Patos } & 15 & -7 & -6 & 4 \\
\text { Galinhas } & 25 & 26 & 18 & 3 \\
\text { Coelhos } & 5 & 19 & 7 & 2 \\
& 4305 & 302 & -109 & 1496 \\
\multicolumn{1}{c}{1^{\mathrm{a}}} & 2^{\mathrm{a}} & 3^{\mathrm{a}} & 4^{\mathrm{a}} & \\
5 & 0 & 0 & 5 & \\
11 & -7 & -6 & 4 \\
22 & 26 & 18 & 3 \\
3 & 19 & 7 & 2 & \\
2809 & 302 & -109 & 1496 \\
1 & 1^{\mathrm{a}} & 2^{\mathrm{a}} & 3^{\mathrm{a}} & 4^{\mathrm{a}} \\
0 & 0 & 0 & 5 \\
7 & -7 & -6 & 4 \\
19 & 26 & 18 & 3 \\
1 & 19 & 7 & 2 \\
1313 & 302 & -109 & 1496
\end{array}
$$

Utilizamos os três passos iniciais, agora entre a $2^{a} \mathrm{e}$ a $3^{a}$ colunas, até eliminarmos o segundo número da $2^{a}$ coluna.

$1^{\mathrm{O}}$ Passo: multiplicamos os termos da $2^{\mathrm{a}}$ coluna $(0,-7,26,19,302)$ pelo $2^{\circ}$ termo da $3^{\mathrm{a}}$ coluna $(-6)$, obtendo $(0,42,-156,-114,-1812)$.

$\begin{array}{ccccc} & 1^{\mathrm{a}} & 2^{\mathrm{a}} & 3^{\mathrm{a}} & 4^{\mathrm{a}} \\ \text { Ovelhas } & 0 & 0 & 0 & 5 \\ \text { Patos } & 7 & 42 & -6 & 4 \\ \text { Galinhas } & 19 & -156 & 18 & 3 \\ \text { Coelhos } & 1 & -114 & 7 & 2 \\ & 1313 & -1812 & -109 & 1496 \\ \\ 1^{\mathrm{a}} & 2^{\mathrm{a}} & 3^{\mathrm{a}} & 4^{\mathrm{a}} & \\ 0 & 0 & 0 & 5 & \\ 7 & 36 & -6 & 4 & \\ \longrightarrow & -138 & 18 & 3 & \\ 19 & -107 & 7 & 2 & \end{array}$
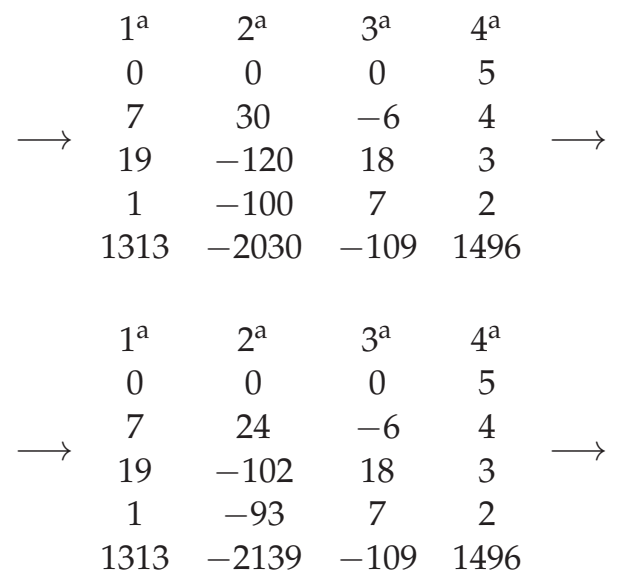

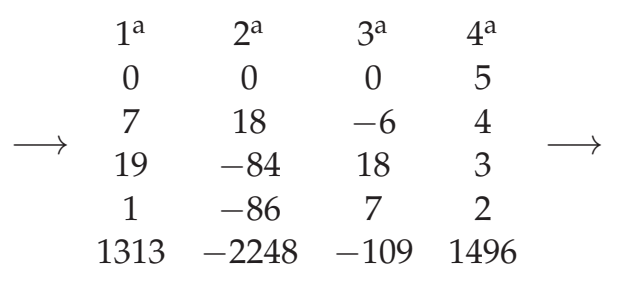

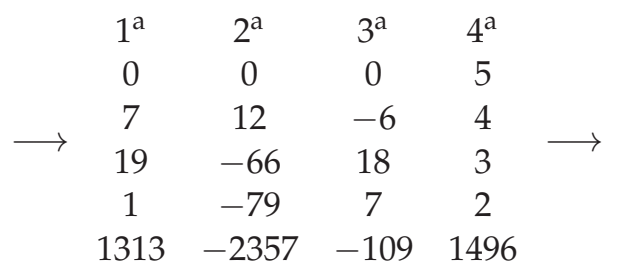

\begin{tabular}{|c|c|c|c|c|}
\hline & $1^{\mathrm{a}}$ & $2^{\mathrm{a}}$ & $3^{\mathrm{a}}$ & $4^{\mathrm{a}}$ \\
\hline Ovelhas & 0 & 0 & 0 & 5 \\
\hline Patos & -42 & 0 & -6 & 4 \\
\hline Galinhas & -114 & -30 & 18 & 3 \\
\hline Coelhos & $\begin{array}{c}-6 \\
-7878\end{array}$ & $\begin{array}{c}-65 \\
-2575\end{array}$ & $\begin{array}{c}7 \\
-109\end{array}$ & $\begin{array}{c}2 \\
1496\end{array}$ \\
\hline
\end{tabular}

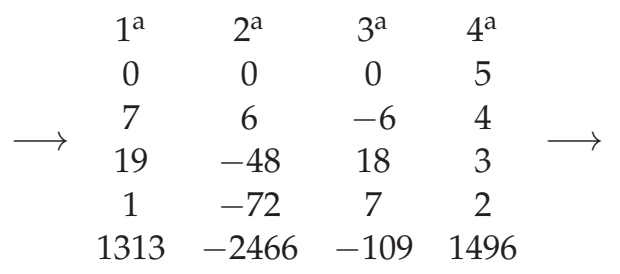

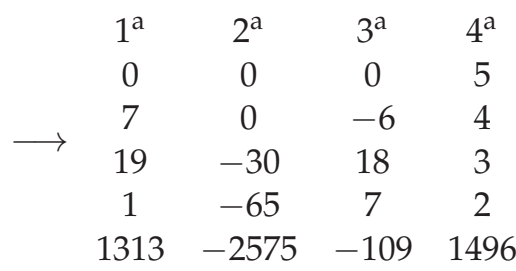

Utilizamos os três passos iniciais, agora entre a $1^{a}$ e $3^{a}$ colunas, até eliminarmos o segundo número da $1^{a}$ coluna.

$1^{\mathrm{o}}$ Passo: multiplicamos os termos da $1^{\mathrm{a}}$ coluna $(0,7,19,1,1313)$ pelo $2^{\circ}$ termo da $3^{\mathrm{a}}$ coluna $(-6)$, obtendose $(0,-42,-114,-6,-7878)$. 


$$
\begin{aligned}
& \begin{array}{ccccc}
1^{\mathrm{a}} & 2^{\mathrm{a}} & 3^{\mathrm{a}} & 4^{\mathrm{a}} & \\
0 & 0 & 0 & 5 & \\
-36 & 0 & -6 & 4 & \\
-132 & -30 & 18 & 3 & \\
-13 & -65 & 7 & 2 & \\
-7769 & -2575 & -109 & 1496 &
\end{array} \longrightarrow
\end{aligned}
$$

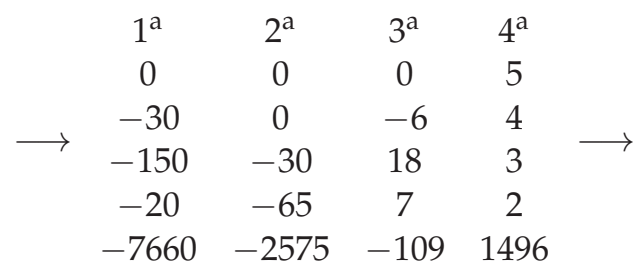

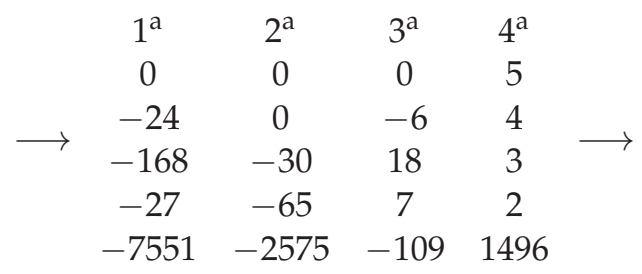

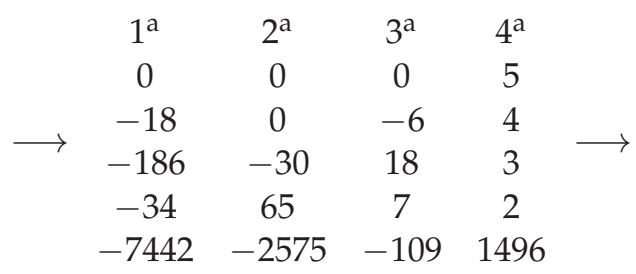

$$
\begin{aligned}
& \begin{array}{ccccc}
1^{\mathrm{a}} & 2^{\mathrm{a}} & 3^{\mathrm{a}} & 4^{\mathrm{a}} & \\
0 & 0 & 0 & 5 & \\
-12 & 0 & -6 & 4 & \\
-204 & -30 & 18 & 3 \\
-41 & -65 & 7 & 2 & \\
-7333 & -2575 & -109 & 1496 & \longrightarrow
\end{array}
\end{aligned}
$$

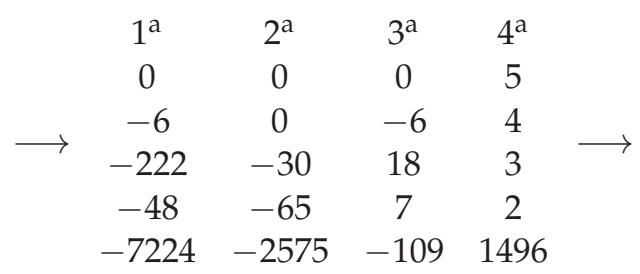

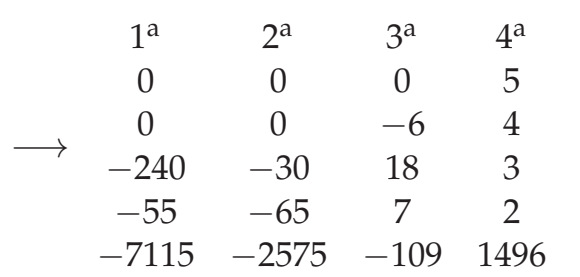

Agora utilizamos os três passos iniciais entre a $1^{a}$ e $2^{a}$ colunas, até eliminarmos o terceiro número da $1^{a}$ coluna.

$1^{\circ}$ Passo: Sabendo que 240 é múltiplo de 30, subtraímos de cada número da $1^{\text {a }}$ coluna o seu correspondente na coluna 2, obtendo em seus lugares:

$(0-0=0 ; 0-0=0 ;-240+30=-210 ;-55+65=$
$10 ;-7115+2575=-4540)$.

$\begin{array}{cccccc} & 1^{\mathrm{a}} & 2^{\mathrm{a}} & 3^{\mathrm{a}} & 4^{\mathrm{a}} & \\ \text { Ovelhas } & 0 & 0 & 0 & 5 & \\ \text { Patos } & 0 & 0 & -6 & 4 & \\ \text { Galinhas } & -210 & -30 & 18 & 3 \\ \text { Coelhos } & 10 & -65 & 7 & 2 & \\ & -4540 & -2575 & -109 & 1496 & \end{array}$

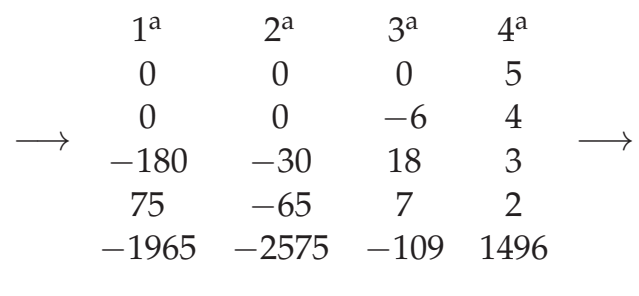

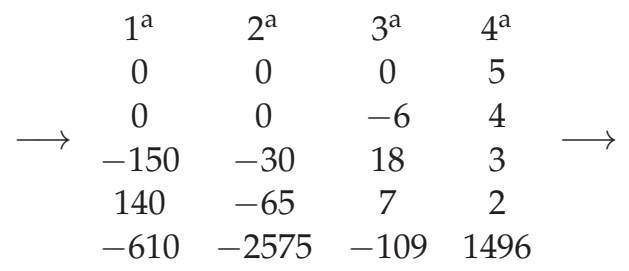

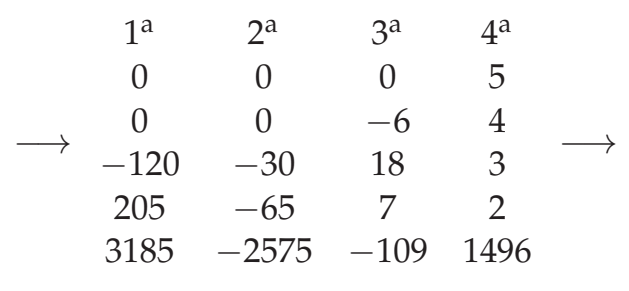$$
\longrightarrow \begin{array}{cccc}
1^{\mathrm{a}} & 2^{\mathrm{a}} & 3^{\mathrm{a}} & 4^{\mathrm{a}} \\
0 & 0 & 0 & 5 \\
0 & 0 & -6 & 4 \\
-90 & -30 & 18 & 3 \\
270 & -65 & 7 & 2 \\
5760 & -2575 & -109 & 1496
\end{array} \longrightarrow
$$

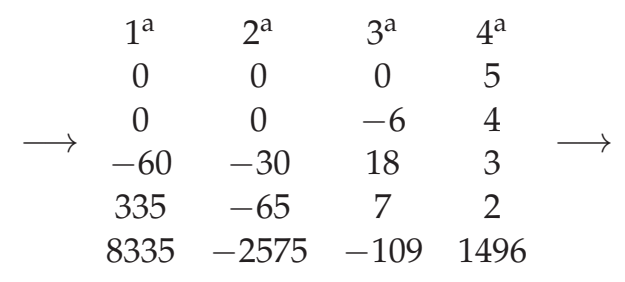

$$
\longrightarrow \begin{array}{ccccc}
1^{\mathrm{a}} & 2^{\mathrm{a}} & 3^{\mathrm{a}} & 4^{\mathrm{a}} & \\
0 & 0 & 0 & 5 & \\
0 & 0 & -6 & 4 & \\
-30 & -30 & 18 & 3 \\
400 & -65 & 7 & 2 & \\
10910 & -2575 & -109 & 1496 & \longrightarrow
\end{array}
$$

$$
\begin{array}{cccc}
1^{\mathrm{a}} & 2^{\mathrm{a}} & 3^{\mathrm{a}} & 4^{\mathrm{a}} \\
0 & 0 & 0 & 5 \\
0 & 0 & -6 & 4 \\
0 & -30 & 18 & 3 \\
465 & -65 & 7 & 2 \\
13485 & -2575 & -109 & 1496
\end{array}
$$


O preço de um coelho é $\frac{13485}{465}$, que equivale a 29 moedas.

O preço de uma galinha é determinado por substituição na coluna que ocupa:

$$
\frac{2575-65 \times 29}{30}=23
$$

Portanto uma galinha vale 23 moedas.

O preço de um pato é determinado por substituição na coluna que ocupa:

$$
\frac{-109-7 \times 29-18 \times 23}{-6}=121
$$

Portanto um pato vale 121 moedas.

O preço de uma ovelha é determinado por substituição na coluna que ocupa:

$$
\frac{1496-2 \times 29-3 \times 23-4 \times 121}{5}=177
$$

Portanto uma ovelha vale 177 moedas.

Nesse problema, atribuindo-se uma letra ao preço de cada animal citado:

a) Preço das ovelhas: $x$.

b) Preço dos patos: $y$.

c) Preço das galinhas: $z$.

d) Preço dos coelhos: $u$.

Temos sua modelagem, via letras, dada pelo conjunto de equações.

$$
\left\{\begin{array}{l}
5 x+4 y+3 z+2 u=1496 \\
4 x+2 y+6 z+3 u=1175 \\
3 x+y+7 z+5 u=958 \\
2 x+3 y+5 z+u=861
\end{array}\right.
$$

Verifiquemos se os valores encontrados realmente satisfazem às equações.

a) Preço das ovelhas: $x=177$ moedas.

b) Preço dos patos: $y=121$ moedas.

c) Preço das galinhas: $z=23$ moedas.

d) Preço dos coelhos: $u=29$ moedas.

$$
\left\{\begin{array}{l}
5 \times 177+4 \times 121+3 \times 23+2 \times 29=1496 \\
4 \times 177+2 \times 121+6 \times 23+3 \times 29=1175 \\
3 \times 177+121+7 \times 23+5 \times 29=958 \\
2 \times 177+3 \times 121+5 \times 23+29=861
\end{array}\right.
$$

A seguir resolvemos dois problemas novos com o objetivo de testar o Método Chinês.

Problema III. Um aluno recebe 3 pontos por problema que acerta e perde 2 pontos por problema que erra. Resolveu 50 problemas e conseguiu 85 pontos. Quantos problemas ele acertou e quantos ele errou?
A modelagem desse problema pode ser feita adotando, para o número de acertos, a letra $x$ e, para o número de erros, a letra $y$. Dessa forma, temos uma igualdade para cada situação descrita, como se segue.

a) Total de questões: $x+y=50$

b) Total de pontos recebidos: $x \times 3$

c) Total de pontos perdidos: $y \times 2$

d) Pontuação final: $3 x-2 y=85$

Em função das letras utilizadas, podemos representar o problema da seguinte forma.

$$
\left\{\begin{array}{c}
x+y=50 \\
3 x-2 y=85
\end{array}\right.
$$

Ilustramos aqui que o método ainda se aplica na eventualidade de trabalharmos com números negativos. Segundo Eves (2004), foram os chineses os primeiros a introduzirem uma notação para números negativos.

Segundo o Método Chinês e usando nosso formato, o problema deve ser representado como a seguir.

$\begin{array}{ccc} & 1^{\mathrm{a}} & 2^{\mathrm{a}} \\ \text { Acertos } & 3 & 1 \\ \text { Erros } & -2 & 1 \\ & 85 & 50\end{array}$

$1^{0}$ Passo: subtraímos o número à direita de cada um dos números da primeira coluna, obtendo $(3-1=$ $2 ;-2-1=-3 ; 85-50=35)$.

$\begin{array}{ccc} & 1^{\mathrm{a}} & 2^{\mathrm{a}} \\ \text { Acertos } & 2 & 1 \\ \text { Erros } & -3 & 1 \\ & 35 & 50\end{array}$

$2^{\circ}$ Passo: subtraímos o número à direita de cada um dos números da primeira coluna, obtendo $(2-1=$ $1 ;-3-1=-4 ; 35-50=-15)$.

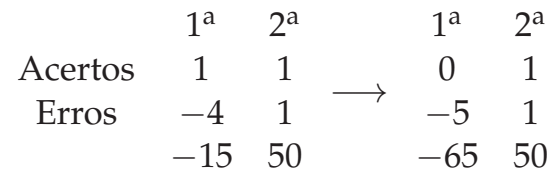

A quantidade de erros é calculada por $\frac{-65}{-5}=13$.

A quantidade de acertos é determinada por substituição.

(n. ${ }^{\circ}$ de acertos $)+13=50 \Rightarrow\left(\right.$ n. ${ }^{\circ}$ de acertos $)=$ $50-13=37$

Verifiquemos se os valores encontrados realmente satisfazem às equações em (1).

$$
\left\{\begin{array}{ccc}
37+13 & =50 \\
3 \times 37-2 \times 13 & =85
\end{array}\right.
$$

Resolvemos outro exemplo, começando com a modelagem por um conjunto de equações e usando, em 
seguida, o método chinês de resolução.

Problema IV. Dona Marianna foi à feira e lá verificou que as barracas da Manuella, da Milena e da Marcella tinham preços diferentes por quilo de produto, conforme a tabela a seguir:

\begin{tabular}{|c||c|c|c|}
\hline & FEIJÃO & ARROZ & FARINHA \\
\hline \hline MANUELLA & $\mathrm{R} \$ 4,00$ & $\mathrm{R} \$ 5,00$ & $\mathrm{R} \$ 3,00$ \\
\hline MILENA & $\mathrm{R} \$ 5,00$ & $\mathrm{R} \$ 4,00$ & $\mathrm{R} \$ 4,00$ \\
\hline MARCELLA & $\mathrm{R} \$ 5,00$ & $\mathrm{R} \$ 4,00$ & $\mathrm{R} \$ 3,00$ \\
\hline
\end{tabular}

Realizando a mesma compra nas barracas da Manuella e da Milena, o gasto será de $R \$ 26,00$. A mesma compra na barraca da Marcella custa $\mathrm{R} \$ 1,00$ a menos. Atribuindo as letras $x, y$ e $z$ às quantidades compradas de feijão, arroz e farinha, respectivamente, qual o valor de $x+y+z$ ?

A modelagem desse problema é feita adotando para a quantidade de $\mathrm{Kg}$ de feijão a letra $x$, para a quantidade de $\mathrm{Kg}$ de arroz a letra $y$ e para a quantidade de $\mathrm{Kg}$ de farinha a letra $z$. Dessa forma, teremos uma igualdade para cada situação, descritas como se segue.

a) Total na barraca da Manuella: $4 x+5 y+3 z=26$

b) Total na barraca da Milena: $5 x+4 y+4 z=26$

c) Total na barraca da Marcella: $5 x+4 y+3 z=25$

Ou seja, o problema pode ser expresso, com a utilização de letras, pelo seguinte conjunto de equações:

$$
\left\{\begin{array}{l}
4 x+5 y+3 z=26 \\
5 x+4 y+4 z=26 \\
5 x+4 y+3 z=25
\end{array}\right.
$$

Segundo o método chinês, o problema deve ser representado da seguinte maneira

$\begin{array}{cccc} & 1^{\mathrm{a}} & 2^{\mathrm{a}} & 3^{\mathrm{a}} \\ \text { Feijão } & 4 & 5 & 5 \\ \text { Arroz } & 5 & 4 & 4 \\ \text { Farinha } & 3 & 4 & 3 \\ & 26 & 26 & 25\end{array}$

$1^{\mathrm{o}}$ Passo: multiplicamos todos os termos da coluna central $(5,4,4,26)$ pelo primeiro termo da coluna à direita (5), obtendo-se $(25,20,20,130)$.

$\begin{array}{cccc} & 1^{\mathrm{a}} & 2^{\mathrm{a}} & 3^{\mathrm{a}} \\ \text { Feijão } & 4 & 25 & 5 \\ \text { Arroz } & 5 & 20 & 4 \\ \text { Farinha } & 3 & 20 & 3 \\ & 26 & 130 & 25\end{array}$

$2^{\circ}$ Passo: subtraímos o número à direita de cada um dos números do centro, obtendo, no centro, $(25-5=$ $20 ; 20-4=16 ; 20-3=17 ; 130-25=105)$.

$\begin{array}{ccccccccc} & 1^{\mathrm{a}} & 2^{\mathrm{a}} & 3^{\mathrm{a}} & & 1^{\mathrm{a}} & 2^{\mathrm{a}} & 3^{\mathrm{a}} & \\ \text { Feijão } & 4 & 20 & 5 & & 4 & 15 & 5 & \\ \text { Arroz } & 5 & 16 & 4 & \longrightarrow & 5 & 12 & 4 & \longrightarrow \\ \text { Farinha } & 3 & 17 & 3 & & 3 & 14 & 3 & \\ & 26 & 105 & 25 & & 26 & 80 & 25 & \end{array}$

$\begin{array}{ccc}1^{\mathrm{a}} & 2^{\mathrm{a}} & 3^{\mathrm{a}} \\ 4 & 10 & 5 \\ \longrightarrow & 8 & 4 \\ 5 & 11 & 3 \\ 26 & 55 & 25\end{array}$

$\begin{array}{cccccccc} & 1^{\mathrm{a}} & 2^{\mathrm{a}} & 3^{\mathrm{a}} & & 1^{\mathrm{a}} & 2^{\mathrm{a}} & 3^{\mathrm{a}} \\ \text { Feijão } & 4 & 5 & 5 & & 4 & 0 & 5 \\ \text { Arroz } & 5 & 4 & 4 & \longrightarrow & 5 & 0 & 4 \\ \text { Farinha } & 3 & 8 & 3 & & 5 & 5 & 3 \\ & 26 & 30 & 25 & & 26 & 5 & 25\end{array}$

Seguimos repetindo os dois primeiros passos, agora entre a $1^{a}$ e $3^{a}$ colunas, eliminando o primeiro elemento da $1^{a}$ coluna.

$1^{\mathrm{O}}$ passo: multiplicar todos os termos da primeira coluna $(4,5,3,26)$ pelo primeiro termo da coluna à direita (5), obtendo-se $(20,25,15,130)$.

$\begin{array}{cccc} & 1^{\mathrm{a}} & 2^{\mathrm{a}} & 3^{\mathrm{a}} \\ \text { Feijão } & 20 & 0 & 5 \\ \text { Arroz } & 25 & 0 & 4 \\ \text { Farinha } & 15 & 5 & 3 \\ & 130 & 5 & 25\end{array}$

$2^{\circ}$ Passo: subtraímos o número à direita de cada um dos números da primeira coluna, obtendo $(20-5=$ $15 ; 25-4=21 ; 15-4=11 ; 130-25=105)$.

\begin{tabular}{|c|c|c|c|c|c|c|c|}
\hline & $1^{\mathrm{a}}$ & $2^{\mathrm{a}}$ & $3^{a}$ & & $1^{\mathrm{a}}$ & $2^{\mathrm{a}}$ & $3^{a}$ \\
\hline Feijão & 15 & 0 & 5 & & 10 & 0 & 5 \\
\hline Arroz & 21 & 0 & 4 & $\longrightarrow$ & . 17 & 0 & 4 \\
\hline \multirow[t]{2}{*}{ Farinha } & 12 & 5 & 3 & & 9 & 5 & 3 \\
\hline & 105 & 5 & 25 & & 80 & 5 & 25 \\
\hline $1^{\mathrm{a}}$ & $2^{a}$ & $3^{\mathrm{a}}$ & & $1^{\mathrm{a}}$ & $2^{\mathrm{a}}$ & $3^{\mathrm{a}}$ & \\
\hline 5 & 0 & 5 & & 0 & 0 & 5 & Feiião \\
\hline \multirow[t]{3}{*}{$\longrightarrow \quad 13$} & 0 & 4 & $\longrightarrow$ & 9 & 0 & 4 & Arroz \\
\hline & 5 & 3 & & 3 & 5 & 3 & Farinha \\
\hline & 5 & 25 & & 30 & 5 & 25 & \\
\hline
\end{tabular}

Podemos observar que a troca de duas colunas dessa tabela não altera a solução proposta pelos chineses, pois estaríamos apenas informando os dados do problema em outra ordem.

A primeira quantidade é $\frac{5}{5}=1$, portanto temos $1 \mathrm{Kg}$ de farinha.

A segunda é determinada por substituição:

$($ Kg de arroz $) \times 9+3 \times 1=30 \Rightarrow($ Kg de arroz $) \times 9+$ $3=30$

$(\mathrm{Kg}$ de arroz $) \times 9=30-3 \Rightarrow(\mathrm{Kg}$ de arroz $) \times 9=27$

$(\mathrm{Kg}$ de arroz $)=\frac{27}{9} \Rightarrow(\mathrm{Kg}$ de arroz $)=3$, portanto são $3 \mathrm{Kg}$ de arroz.

A terceira, também por substituição:

$(\mathrm{Kg}$ de feijão $) \times 5+4 \times 3+3 \times 1=25 \Rightarrow(\mathrm{Kg}$ de feijão) $\times 5+12+3=25$

$($ Kg de feijão $) \times 5+15=25 \Rightarrow($ Kg de feijão $) \times 5=10$ 
$(\mathrm{Kg}$ de feijão $)=\frac{10}{5} \Rightarrow(\mathrm{Kg}$ de feijão $)=2$, portanto são $2 \mathrm{Kg}$ de feijão.

Verificamos se os valores encontrados realmente satisfazem às equações.

Temos sua modelagem, via letras, dada por:

$$
\left\{\begin{array}{l}
4 x+5 y+3 z=26 \\
5 x+4 y+4 z=26 \\
5 x+4 y+3 z=25
\end{array}\right.
$$

a) Quantidade de feijão: $x=2$.

b) Quantidade de arroz: $y=3$.

c) Quantidade de farinha: $z=1$.

$$
\left\{\begin{array}{l}
4 \times 2+5 \times 3+3 \times 1=26 \\
5 \times 2+4 \times 3+4 \times 1=26 \\
5 \times 2+4 \times 3+3 \times 1=25
\end{array}\right.
$$

É possível perceber que os valores numéricos apresentados na vertical em "Os nove capítulos da arte matemática"aparecem horizontalmente na modelagem em que usamos letras para representar quantidades (nas equações).

Então vemos que podemos usar o Método Chinês para encontrar os valores das letras de uma dada quantidade de equações.

Formalizemos alguns conceitos vistos até o momento.

A utilização de letras representando quantidades nos leva a uma modelagem por equações da forma a seguir.

$$
a_{1} \cdot x_{1}+a_{2} \cdot x_{2}+\cdots+a_{n} \cdot x_{n}=b
$$

Essa forma é chamada de equação linear, na qual:

- $x_{1}, x_{2}, \cdots, x_{n}$ são chamados de incógnitas, que são as letras que aparecem na equação;

- $a_{1}, a_{2}, \cdots, a_{n}$ são chamados de coeficientes das incógnitas, sendo os números que aparecem multiplicando as incógnitas na equação;

- $b$ é o termo independente, sendo o número que aparece sem incógnitas na equação.

Uma solução da equação (1) é uma n-upla ${ }^{1}\left(\alpha_{1}, \alpha_{2}, \cdots, \alpha_{n}\right)$, de tal modo que, quando substituímos, na equação, $x_{1}$ por $\alpha_{1}, x_{2}$ por $\alpha_{2}, \cdots, x_{n}$ por $\alpha_{n}$, tal equação é satisfeita, ou seja,

$$
a_{1} \cdot \alpha_{1}+a_{2} \cdot \alpha_{2}+\cdots+a_{n} \cdot \alpha_{n}=b
$$

Chamamos sistema de equações lineares o conjunto de duas ou mais equações lineares com as mesmas incógnitas.

\footnotetext{
${ }^{1}$ n-upla: sequência ordenada de números que se referem a $n$ variáveis.
}

$\left\{\begin{array}{ccccccccc}a_{11} \cdot x_{1} & + & a_{12} \cdot x_{2} & + & \cdots & + & a_{1 n} \cdot x_{n} & = & b_{1} \\ a_{21} \cdot x_{1} & + & a_{22} \cdot x_{2} & + & \cdots & + & a_{2 n} \cdot x_{n} & = & b_{2} \\ \vdots & & \vdots & & \ddots & & \vdots & & \vdots \\ a_{m 1} \cdot x_{1} & + & a_{m 2} \cdot x_{2} & + & \cdots & + & a_{m n} \cdot x_{n} & = & b_{m}\end{array}\right.$

Uma solução para esse sistema é a n-upla $\left(\alpha_{1}, \alpha_{2}, \cdots, \alpha_{n}\right)$, que satisfaz simultaneamente a todas as equações envolvidas.

$$
\left\{\begin{array}{ccccccccc}
a_{11} \cdot \alpha_{1} & + & a_{12} \cdot \alpha_{2} & + & \cdots & + & a_{1 n} \cdot \alpha_{n} & = & b_{1} \\
a_{21} \cdot \alpha_{1} & + & a_{22} \cdot \alpha_{2} & + & \cdots & + & a_{2 n} \cdot \alpha_{n} & = & b_{2} \\
\vdots & & \vdots & & \ddots & & \vdots & & \vdots \\
a_{m 1} \cdot \alpha_{1} & + & a_{m 2} \cdot \alpha_{2} & + & \cdots & + & a_{m n} \cdot \alpha_{n} & = & b_{m}
\end{array}\right.
$$

Diante disso, podemos perceber que os problemas (I), (II), (III) e (IV), que resolvemos, são sistemas lineares aos quais aplicamos o método de resolução dos chineses.

\section{Porque o método chinês funciona}

Nesta seção formalizamos as operações utilizadas no método chinês e verificamos que elas possuem propriedades bem interessantes. O método, como já foi dito, foi utilizado apenas para resolver sistemas em que o número de equações era igual ao número de incógnitas, entretanto essas operações podem ser aplicadas na resolução de sistemas com $m$ equações e $n$ incógnitas, para $m$ e $n$ quaisquer.

Definimos a seguir, formalmente, as operações utilizadas pelos chineses, que são chamadas de operações elementares, mostrando que tais operações, quando aplicadas nas equações de um sistema linear, o transformam em outro sistema, chamado sistema equivalente, cujas soluções são as mesmas do sistema inicial.

Ilustramos as operações elementares em um sistema de 3 equações e 4 incógnitas, mas essas operações continuam valendo para um sistema de $m$ equações e $n$ incógnitas, com $m$ e $n$ quaisquer:

\section{Operação Elementar 1 - Permutar uma ou mais equa-} ções

Notemos que, se $\left(\alpha_{1}, \alpha_{2}, \alpha_{3}, \alpha_{4}\right)$ é solução do sistema

$(A)\left\{\begin{array}{l}a_{11} x_{1}+a_{12} x_{2}+a_{13} x_{3}+a_{14} x_{4}=b_{1} \\ a_{21} x_{1}+a_{22} x_{2}+a_{23} x_{3}+a_{24} x_{4}=b_{2} \\ a_{31} x_{1}+a_{32} x_{2}+a_{33} x_{3}+a_{34} x_{4}=b_{3}\end{array}\right.$,

então ela também será solução do sistema obtido pela troca de lugar entre duas linhas e, também pelo mesmo motivo, a recíproca será verdadeira. Em outras palavras, trocar a ordem das equações não muda o problema em si, nem a sua solução, apenas corresponde a descrever o problema em uma ordem diferente, logo a solução continuará a mesma. 
Operação Elementar 2 - Somar os termos correspondentes de duas equações e colocar o resultado em substituição a uma das equações envolvidas na operação

Notemos que, se $\left(\alpha_{1}, \alpha_{2}, \alpha_{3}, \alpha_{4}\right)$ é solução do sistema $(A)$, permanecerá solução do sistema a seguir.

$$
(B)\left\{\begin{array}{l}
a_{11} x_{1}+a_{12} x_{2}+a_{13} x_{3}+a_{14} x_{4}=b_{1} \\
\left(a_{11}+a_{21}\right) x_{1}+\left(a_{12}+a_{22}\right) x_{2}+ \\
+\left(a_{13}+a_{23}\right) x_{3}+\left(a_{14}+a_{24}\right) x_{4}=b_{1}+b_{2} \\
a_{31} x_{1}+a_{32} x_{2}+a_{33} x_{3}+a_{34} x_{4}=b_{3}
\end{array}\right.
$$

De fato, sabemos que todas as equações, exceto a $2^{a}$, continuam sendo as mesmas, logo uma solução do sistema $(A)$ satisfaria a todas as equações do sistema $(B)$, exceto a $2^{a}$, mas mostraremos que essa equação também é satisfeita.

Da $1^{a}$ equação em $(A)$, temos $a_{11} \alpha_{1}+a_{12} \alpha_{2}+a_{13} \alpha_{3}+$ $a_{14} \alpha_{4}=b_{1}$ e da $2^{a}$ equação temos $a_{21} \alpha_{1}+a_{22} \alpha_{2}+a_{23} \alpha_{3}+$ $a_{24} \alpha_{4}=b_{2}, \log$, somando as equações e colocando os termos iguais em evidência, temos $\left(a_{11}+a_{21}\right) \alpha_{1}+\left(a_{12}+\right.$ $\left.a_{22}\right) \alpha_{2}+\left(a_{13}+a_{23}\right) \alpha_{3}+\left(a_{14}+a_{24}\right) \alpha_{4}=b_{1}+b_{2}$, ou seja, $\left(\alpha_{1}, \alpha_{2}, \alpha_{3}, \alpha_{4}\right)$ satisfaz à segunda equação de $(B)$.

Reciprocamente, mostraremos que, se $\left(\alpha_{1}, \alpha_{2}, \alpha_{3}, \alpha_{4}\right)$ satisfaz às equações do sistema $(B)$, também satisfaz às equações do sistema $(A)$.

$$
\left\{\begin{array}{l}
a_{11} \alpha_{1}+a_{12} \alpha_{2}+a_{13} \alpha_{3}+a_{14} \alpha_{4}=b_{1} \\
\left(a_{11}+a_{21}\right) \alpha_{1}+\left(a_{12}+a_{22}\right) \alpha_{2}+ \\
+\left(a_{13}+a_{23}\right) \alpha_{3}+\left(a_{14}+a_{24}\right) \alpha_{4}=b_{1}+b_{2} \\
a_{31} x_{1}+a_{32} \alpha_{2}+a_{33} \alpha_{3}+a_{34} \alpha_{4}=b_{3}
\end{array}\right.
$$

então

$$
\left\{\begin{array}{l}
a_{11} \alpha_{1}+a_{12} \alpha_{2}+a_{13} \alpha_{3}+a_{14} \alpha_{4}=b_{1} \\
a_{11} \alpha_{1}+a_{21} \alpha_{1}+a_{12} \alpha_{2}+a_{22} \alpha_{2}+ \\
+a_{13} \alpha_{3}+a_{23} \alpha_{3}+a_{14} \alpha_{4}+a_{24} \alpha_{4}=b_{1}+b_{2} \\
a_{31} \alpha_{1}+a_{32} \alpha_{2}+a_{33} \alpha_{3}+a_{34} \alpha_{4}=b_{3}
\end{array}\right.
$$

Da primeira equação temos $a_{11} \alpha_{1}+a_{12} \alpha_{2}+a_{13} \alpha_{3}+$ $a_{14} \alpha_{4}=b_{1}$, então a segunda equação do sistema anterior corresponde a $b_{1}+a_{21} \alpha_{1}+a_{22} \alpha_{2}+a_{23} \alpha_{3}+a_{24} \alpha_{4}=b_{1}+$ $b_{2}$, ou ainda a $a_{21} \alpha_{1}+a_{22} \alpha_{2}+a_{23} \alpha_{3}+a_{24} \alpha_{4}=b_{2}$. Dessa forma, o sistema acima pode ser reescrito como:

$$
\left\{\begin{array}{l}
a_{11} \alpha_{1}+a_{12} \alpha_{2}+a_{13} \alpha_{3}+a_{14} \alpha_{4}=b_{1} \\
a_{21} \alpha_{1}+a_{22} \alpha_{2}+a_{23} \alpha_{3}+a_{24} \alpha_{4}=b_{2} \\
a_{31} \alpha_{1}+a_{32} \alpha_{2}+a_{33} \alpha_{3}+a_{34} \alpha_{4}=b_{3}
\end{array}\right.
$$

$\operatorname{Logo}\left(\alpha_{1}, \alpha_{2}, \alpha_{3}, \alpha_{4}\right)$ é solução de $(A)$.

Operação Elementar 3 - Multiplicar uma ou mais equações por escalares reais diferentes de zero

- Observemos que, se $\left(\alpha_{1}, \alpha_{2}, \alpha_{3}, \alpha_{4}\right)$ é solução do sistema $(A)$, então será solução do sistema

$$
(C)\left\{\begin{array} { l l l } 
{ \beta a _ { 1 1 } x _ { 1 } + \beta a _ { 1 2 } x _ { 2 } + \beta a _ { 1 3 } x _ { 3 } + \beta a _ { 1 4 } x _ { 4 } = \beta b _ { 1 } } \\
{ a _ { 2 1 } x _ { 1 } + a _ { 2 2 } x _ { 2 } + a _ { 2 3 } x _ { 3 } + a _ { 2 4 } x _ { 4 } = b _ { 2 } } \\
{ a _ { 3 1 } x _ { 1 } + a _ { 3 2 } x _ { 2 } + a _ { 3 3 } x _ { 3 } + a _ { 3 4 } x _ { 4 } = b _ { 3 } }
\end{array} \left\{\begin{array}{l}
\beta a_{11} x_{1}+\beta a_{12} x_{2}+\beta a_{13} x_{3}+\beta a_{14} x_{4}=\beta b_{1} \\
\left(\beta a_{13}+\gamma a_{21}\right) x_{1}+\left(\beta a_{12}+\gamma a_{22}\right) x_{2}+ \\
+\left(\beta a_{13}+\gamma a_{23}\right) x_{3}+\left(\beta a_{14}+\gamma a_{24}\right) x_{4}=\beta b_{1}+\gamma b_{2} \\
a_{31} x_{1}+a_{32} x_{2}+a_{33} x_{3}+a_{34} x_{4}=b_{3}
\end{array}\right.\right.
$$

Se $\left(\alpha_{1}, \alpha_{2}, \alpha_{3}, \alpha_{4}\right)$ satisfaz a $(A)$, então já satisfaz a todas as equações de $(C)$, exceto à primeira. Mostraremos, no entanto, que $\left(\alpha_{1}, \alpha_{2}, \alpha_{3}, \alpha_{4}\right)$ também satisfaz à $1^{a}$ equação.

De $(A)$, temos que $a_{11} x_{1}+a_{12} x_{2}+a_{13} x_{3}+a_{14} x_{4}=b_{1}$ , logo, multiplicando tudo por $\beta$, com $\beta \neq 0$, temos $\beta a_{11} x_{1}+\beta a_{12} x_{2}+\beta a_{13} x_{3}+\beta a_{14} x_{4}=\beta b_{1}$, então satisfaz à $1^{a}$ equação de $(C)$.

Reciprocamente, mostraremos que, se $\left(\alpha_{1}, \alpha_{2}, \alpha_{3}, \alpha_{4}\right)$ satisfaz ao sistema $(C)$, também satisfaz ao sistema $(A)$.

$$
\begin{aligned}
& \left\{\begin{array}{l}
\beta a_{11} x_{1}+\beta a_{12} x_{2}+\beta a_{13} x_{3}+\beta a_{14} x_{4}=\beta b_{1} \\
a_{21} x_{1}+a_{22} x_{2}+a_{23} x_{3}+a_{24} x_{4}=b_{2} \\
a_{31} x_{1}+a_{32} x_{2}+a_{33} x_{3}+a_{34} x_{4}=b_{3}
\end{array}\right. \\
& \Rightarrow\left\{\begin{array}{llll}
\beta\left(a_{11} x_{1}+a_{12} x_{2}+a_{13} x_{3}+a_{14} x_{4}\right) & =\beta b_{1} \\
a_{21} x_{1}+a_{22} x_{2}+a_{23} x_{3}+a_{24} x_{4} & =b_{2} \\
a_{31} x_{1}+a_{32} x_{2}+a_{33} x_{3}+a_{34} x_{4} & =b_{3}
\end{array}\right.
\end{aligned}
$$

Como $\beta \neq 0$, dividiremos os dois lados da $1^{a}$ equação por $\beta$.

$\Rightarrow\left\{\begin{array}{l}a_{11} x_{1}+a_{12} x_{2}+a_{13} x_{3}+a_{14} x_{4}=b_{1} \\ a_{21} x_{1}+a_{22} x_{2}+a_{23} x_{3}+a_{24} x_{4}=b_{2} \\ a_{31} x_{1}+a_{32} x_{2}+a_{33} x_{3}+a_{34} x_{4}=b_{3}\end{array}\right.$

Logo $\left(\alpha_{1}, \alpha_{2}, \alpha_{3}, \alpha_{4}\right)$ é solução de $(A)$.

Operação Elementar 4 - A uma equação adicionar outra, multiplicada por um escalar diferente de zero, e colocar o resultado em substituição a uma das equações envolvidas na operação

Suponhamos que $\left(\alpha_{1}, \alpha_{2}, \alpha_{3}, \alpha_{4}\right)$ seja solução do sistema $(A)$.

Então também será solução do sistema a seguir, pela propriedade III, com $\beta \neq 0$ e $\gamma \neq 0$.

$$
\left\{\begin{array}{l}
\beta a_{11} x_{1}+\beta a_{12} x_{2}+\beta a_{13} x_{3}+\beta a_{14} x_{4}=\beta b_{1} \\
\gamma a_{21} x_{1}+\gamma a_{22} x_{2}+\gamma a_{23} x_{3}+\gamma a_{24} x_{4}=\gamma b_{2} \\
a_{31} x_{1}+a_{32} x_{2}+a_{33} x_{3}+a_{34} x_{4}=b_{3}
\end{array}\right.
$$

E também será solução do sistema a seguir, pela propriedade II.

$$
\left\{\begin{array}{l}
\beta a_{11} x_{1}+\beta a_{12} x_{2}+\beta a_{13} x_{3}+\beta a_{14} x_{4}=\beta b_{1} \\
\left(\beta a_{11}+\gamma a_{21}\right) x_{1}+\left(\beta a_{12}+\gamma a_{22} x_{2}\right)+ \\
+\left(\beta a_{13}+\gamma a_{23}\right) x_{3}+\left(\beta a_{14}+\gamma a_{24}\right) x_{4}=\beta b_{1}+\gamma b_{2} \\
a_{31} x_{1}+a_{32} x_{2}+a_{33} x_{3}+a_{34} x_{4}=b_{3}
\end{array}\right.
$$

Reciprocamente, se $\left(\alpha_{1}, \alpha_{2}, \alpha_{3}, \alpha_{4}\right)$ é solução do sistema: 
Ou seja,

$$
\left\{\begin{array}{l}
\beta a_{11} \alpha_{1}+\beta a_{12} \alpha_{2}+\beta a_{13} \alpha_{3}+\beta a_{14} \alpha_{4}=\beta b_{1} \\
\beta a_{11} \alpha_{1}+\gamma a_{21} \alpha_{1}+\beta a_{12} \alpha_{2}+\gamma a_{22} \alpha_{2}+ \\
+\beta a_{13} \alpha_{3}+\gamma a_{23} \alpha_{3}+\beta a_{14} \alpha_{4}+\gamma a_{24} \alpha_{4}=\beta b_{1}+\gamma b_{2} \\
a_{31} \alpha_{1}+a_{32} \alpha_{2}+a_{33} \alpha_{3}+a_{34} \alpha_{4}=b_{3}
\end{array}\right.
$$

Na primeira equação temos $\beta a_{11} \alpha_{1}+\beta a_{12} \alpha_{2}+\beta a_{13} \alpha_{3}+$ $\beta a_{14} \alpha_{4}=\beta b_{1}$, então a segunda equação do sistema anterior corresponde a $\beta b_{1}+\gamma a_{21} \alpha_{1}+\gamma \gamma a_{22} \alpha_{2}+\gamma a_{23} \alpha_{3}+$ $\gamma a_{24} \alpha_{4}=\beta b_{1}+\gamma b_{2}$, ou, ainda, a $\gamma a_{21} \alpha_{1}++\gamma a_{22} \alpha_{2}+$ $\gamma a_{23} \alpha_{3}+\gamma a_{24} \alpha_{4}=\gamma b_{2}$. Dessa forma, o sistema acima pode ser reescrito como

$\left\{\begin{array}{l}\beta a_{11} \alpha_{1}+\beta a_{12} \alpha_{2}+\beta a_{13} \alpha_{3}+\beta a_{14} \alpha_{4}=\beta b_{1} \\ \gamma a_{21} \alpha_{1}+\gamma a_{22} \alpha_{2}+\gamma a_{23} \alpha_{3}+\gamma a_{24} \alpha_{4}=\gamma b_{2} \\ a_{31} \alpha_{1}+a_{32} \alpha_{2}+a_{33} \alpha_{3}+a_{34} \alpha_{4}=b_{3}\end{array}\right.$

Então, temos

$\left\{\begin{array}{c}\beta\left(a_{11} \alpha_{1}+a_{12} \alpha_{2}+a_{13} \alpha_{3}+a_{14} \alpha_{4}\right)=\beta b_{1} \\ \gamma\left(a_{21} \alpha_{1}+a_{22} \alpha_{2}+a_{23} \alpha_{3}+a_{24} \alpha_{4}\right)=\gamma b_{2} \\ a_{31} \alpha_{1}+a_{32} \alpha_{2}+a_{33} \alpha_{3}+a_{34} \alpha_{4}=b_{3}\end{array}\right.$

Como $\beta \neq 0$ e $\gamma \neq 0$, dividiremos os dois lados da primeira equação por $\beta$ e os dois lados da segunda equação por $\gamma$, obtendo

$$
\left\{\begin{array}{l}
a_{11} \alpha_{1}+a_{12} \alpha_{2}+a_{13} \alpha_{3}+a_{14} \alpha_{4}=b_{1} \\
a_{21} \alpha_{1}+a_{22} \alpha_{2}+a_{23} \alpha_{3}+a_{24} \alpha_{4}=b_{2} \\
a_{31} \alpha_{1}+a_{32} \alpha_{2}+a_{33} \alpha_{3}+a_{34} \alpha_{4}=b_{3}
\end{array}\right.
$$

Logo, $\left(\alpha_{1}, \alpha_{2}, \alpha_{3}, \alpha_{4}\right)$ é solução do nosso sistema $(A)$.

Notemos que, nos problemas (III) e (IV), apresentados no início deste capítulo, bem como nos problemas (I) e (II), propostos pelos chineses, o número de incógnitas é igual ao número de equações, assim, generalizando o modelo, indicando cada linha e cada coluna em que aparece um elemento com seu coeficiente, temos o sistema a seguir:

$$
\left\{\begin{array}{ccccccccc}
a_{11} \cdot x_{1} & + & a_{12} \cdot x_{2} & + & \cdots & + & a_{1 n} \cdot x_{n} & = & b_{1} \\
a_{21} \cdot x_{1} & + & a_{22} \cdot x_{2} & + & \cdots & + & a_{2 n} \cdot x_{n} & = & b_{2} \\
\vdots & & \vdots & & \ddots & & \vdots & & \vdots \\
a_{n 1} \cdot x_{1} & + & a_{n 2} \cdot x_{2} & + & \cdots & + & a_{n n} \cdot x_{n} & = & b_{n}
\end{array}\right.
$$

Em um sistema $n \times n$, no caso de não nos depararmos com uma igualdade absurda ou uma identidade, o método dos chineses encontra o valor para $x_{n}$, em seguida para $x_{n-1}, \cdots, x_{1}$, um por um, através de substituições sucessivas. Esse tipo de cálculo foi realizado pelo dispositivo chinês chamado placa de contagem.

Para um sistema $3 \times 3$, como

$$
\left\{\begin{array}{l}
a_{11} x_{1}+a_{12} x_{2}+a_{13} x_{3}=b_{1} \\
a_{21} x_{1}+a_{22} x_{2}+a_{23} x_{3}=b_{2} \\
a_{31} x_{1}+a_{32} x_{2}+a_{33} x_{3}=b_{3}
\end{array}\right.
$$

realizamos operações elementares, visando a encontrar um sistema equivalente com a forma

$$
\left\{\begin{aligned}
a_{11}^{\prime} x_{1}+a_{12}^{\prime} x_{2}+a_{13}^{\prime} x_{3} & =b_{1}^{\prime} \\
a_{22}^{\prime} x_{2}+a_{23}^{\prime} x_{3} & =b_{2}^{\prime} \\
a_{33}^{\prime} x_{3} & =b_{3}^{\prime}
\end{aligned}\right.
$$

que é equivalente ao método chinês sair de

$\begin{array}{lll}a_{31} & a_{21} & a_{11} \\ a_{32} & a_{22} & a_{12} \\ a_{33} & a_{23} & a_{13} \\ b_{3} & b_{2} & b_{1}\end{array}$

e chegar a

$$
\begin{array}{ccc}
0 & 0 & a_{11}^{\prime} \\
0 & a_{22}^{\prime} & a_{12}^{\prime} \\
a_{33}^{\prime} & a_{23}^{\prime} & a_{13}^{\prime} \\
b_{3}^{\prime} & b_{2}^{\prime} & b_{1}^{\prime}
\end{array}
$$

A seguir, fazemos as operações aprendidas pelo método chinês diretamente no sistema. Para ficar claro como tais operações são realizadas, exemplificamos em um sistema com duas equações e duas incógnitas $(2 \times 2)$ e em outro com três equações e três incógnitas $(3 \times 3)$. Em sistemas maiores, os passos são os mesmos.

Exemplo 1 (FUVEST 1994 - adaptada) Aline diz a Antonia: se você me der $\frac{1}{5}$ do dinheiro que possui, eu ficarei com uma quantia igual ao dobro do que lhe restará. Por outro lado, se eu lhe der $\mathrm{R} \$ 6.000,00$ do meu dinheiro, nós ficaremos com quantias iguais. Quanto dinheiro possui cada uma?

Na modelagem desse problema a incógnita $x$ representa a quantia que Aline possui e a incógnita $y$ a quantia que Antônia possui, $\mathrm{O}$ problema nos informa que:

a) Antonia dá $\frac{1}{5}$ do dinheiro que tem: $y-\frac{1 y}{5}$

b) Aline recebe $\frac{1}{5}$ do dinheiro de Antonia: $x+\frac{1 y}{5}$

c) Aline fica com o dobro do que resta a Antonia: $x+\frac{y}{5}=2\left(y-\frac{y}{5}\right)$

d) Aline dá R\$ 6.000,00 a Antonia: $x-6000$

e) Antonia recebe $\mathrm{R} \$ 6.000,00$ de Aline: $y+6000$

f) Aline e Antonia ficam com quantias iguais: $x-$ $6000=y+6000$

Essas informações podem ser representadas pelo seguinte sistema

$$
\begin{aligned}
& \left\{\begin{aligned}
2\left(y-\frac{y}{5}\right) & =x+\frac{y}{5} \\
x-6000 & =y+6000
\end{aligned}\right.
\end{aligned}
$$

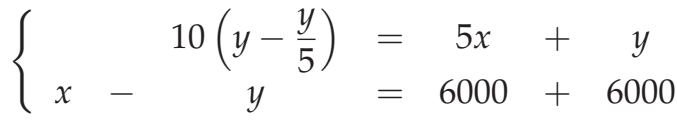

$$
\begin{aligned}
& \left\{\begin{array}{ccc}
10 y-2 y & =5 x+y \\
x-y & =12000
\end{array}\right.
\end{aligned}
$$




$$
\begin{aligned}
& \left\{\begin{aligned}
8 y & =5 x \\
x-y & =12000
\end{aligned}\right. \\
& \left\{\begin{array}{ccc}
5 x-7 y & = & 0 \\
x-y & = & 12000
\end{array}\right.
\end{aligned}
$$

Resolvemos esse sistema usando as operações elementares já vistas.

$$
\left\{\begin{array}{cccc}
5 x-7 y & = & 0 \\
x-y & = & 12000
\end{array}\right.
$$

Trocando as posições das equações

$$
\left\{\begin{array}{ccc}
x-y & = & 12000 \\
5 x-7 y & =0
\end{array}\right.
$$

Anulando a $1^{a}$ incógnita da $2^{a}$ equação. Isso é possível pelo uso das operações elementares apresentadas anteriormente. Substituiremos a segunda equação pela diferença entre 5 vezes a primeira equação e a segunda equação. O lado esquerdo da nova equação é dado por

$$
5(x-y)-(5 x-7 y)=2 y
$$

e o lado direito

$$
5.12000-0=60000
$$

e a equação que substituirá a segunda equação é

$$
\begin{gathered}
2 y=60000 . \\
\left\{\begin{aligned}
x-y & =12000 \\
2 y & =60000 \Rightarrow y=30000
\end{aligned}\right.
\end{gathered}
$$

Substituindo a $2^{a}$ incógnita na $1^{a}$ equação

$$
x-30000 y=12000 \Rightarrow x=420000 .
$$

Verifiquemos se os valores encontrados realmente satisfazem às equações.

Temos sua modelagem, via letras, dada por

$$
\left\{\begin{array}{cccc}
5 x-7 y & = & 0 \\
x-y & = & 12000
\end{array}\right.
$$

a) Para a quantia de Aline, $x=42000$.

b) Para a quantia de Antonia, $y=30000$.

$$
\begin{cases}5 \times 42000-7 \times 30000 & =0 \\ 42000-30000 & =12000\end{cases}
$$

Respondendo à pergunta, Aline possui $\mathrm{R} \$ 42.000,00 \mathrm{e}$ Antônia possui $\mathrm{R} \$ 30.000,00$.

Exemplo 2 Um casal entrou em uma lanchonete e pediu 3 sanduíches, 1 refrigerante e 2 sobremesas, gastando $\mathrm{R} \$ 43,00$. Na mesa ao lado, algumas pessoas pediram 8 sanduíches, 3 refrigerantes e 5 sobremesas, gastando $\mathrm{R} \$ 114,00$. O preço de 1 sanduíche, mais o de 1 refrigerante, mais o de 1 sobremesa totaliza $\mathrm{R} \$ 20,00$. Qual o preço de cada um desses itens?

A modelagem desse problema foi feita, adotando para o preço do sanduíche, a incógnita $x$; para o preço do refrigerante, a incógnita $y$; e, para o preço da sobremesa, a incógnita $z$. As informações dadas podem ser escritas da seguinte forma:

a) 1 sanduíche, 1 refrigerante e 1 sobremesa custam $\mathrm{R} \$ 20,00$ :

$$
x+y+z=20 .
$$

b) 8 sanduíches, 3 refrigerantes e 5 sobremesas custam $\mathrm{R} \$ 114,00$ :

$$
8 x+3 y+5 z=114 .
$$

c) 3 sanduíches, 1 refrigerante e 2 sobremesas custam $\mathrm{R} \$ 43,00$ :

$$
3 x+y+2 z=43 .
$$

O sistema a seguir descreve o problema:

$$
\left\{\begin{array}{c}
x+y+z=20 \\
8 x+3 y+5 z=114 \\
3 x+y+2 z=43
\end{array}\right.
$$

Anulemos a primeira incógnita da segunda e terceira equações. Para anular na segunda equação, substituímos a segunda equação pela diferença entre 8 vezes a primeira equação e a segunda equação. Com isso obtemos do lado esquerdo da equação igual a $8(x+y+z)-(8 x+3 y+5 z)=5 y+3 z$ e o lado direito igual a $8.20-114=46$ e o sistema equivalente fica

$$
\left\{\begin{aligned}
x+y+z & =20 \\
5 y+3 z & =46 \\
3 x+y+2 z & =43
\end{aligned}\right.
$$

Para anular na terceira equação, substituímos a terceira equação pela diferença entre 3 vezes a primeira equação e a terceira equação. Com isso obtemos do lado esquerdo da equação igual a $3(x+y+z)-(3 x+y+2 z)=2 y+$ $z$ e o lado direito igual a $3.20-43=17$ e o sistema equivalente fica

$$
\left\{\begin{aligned}
x+y+z & =20 \\
5 y+3 z & =46 \\
2 y+z & =17
\end{aligned}\right.
$$

Agora anulamos a segunda incógnita na terceira equação. Para isso substitua a terceira equação pela diferença entre 2 vezes a segunda equação e 5 vezes a terceira equação. Assim o lado esquerdo da equação é $2(5 y+3 z)-5(2 y+z)=z$ e o lado direito $2.46-5.17=$ 7 e o sistema equivalente fica:

$$
\left\{\begin{aligned}
x+y+z & =20 \\
5 y+3 z & =46 \\
z & =7
\end{aligned}\right.
$$


Neste último sistema, a solução encontrada corresponde à mesma do sistema original, mas apresenta um grau de dificuldade menor, no que diz respeito às operações de substituições envolvidas.

Substituindo na segunda equação o valor de $z=7$, obtido na terceira equação, temos

$5 y+3.7=46 \Rightarrow 5 y+21=46 \Rightarrow 5 y=25 \Rightarrow y=5$.

Substituindo, na primeira equação, os valores de $z$ e $y$ obtidos anteriormente, temos,

$$
x+5+7=20 \Rightarrow x+12=20 \Rightarrow x=8
$$

Verifiquemos se os valores encontrados realmente satisfazem às equações de (3)

$$
\left\{\begin{array}{c}
8+5+7=20 \\
8 \times 8+3 \times 5+5 \times 7=114 \\
3 \times 8+5+2 \times 7=43
\end{array}\right.
$$

Respondendo à pergunta, um sanduíche custa $R \$ 8,00$, um refrigerante custa $R \$ 5,00$ e uma sobremesa custa $\mathrm{R} \$ 7,00$.

O Método Chinês funcionava, por que todos os problemas que eles tentavam resolver utilizando tal método admitia uma única solução, ou seja, os problemas proposto para ser resolvido pelo método eram na linguagem de hoje, possíveis determinados.

\section{Referências}

Carrera, J. P. i. (2009). Liu Hui: Nueve Capitulos de Las Matematicas CHINAS. Nivola Libros Y Ediciones, S.L., Madrid..

Dante, L. R. (2010). Matemática: Contexto e Aplicações, vol. 2. São Paulo: Ática.

Eves, H. (2004). Introdução à História da Matemática. Campinas, SP: Editora da UNICAMP.

Ferrari, S. L. P., Cribari-Neto, F. (2004). Beta regression for modelling rates and proportions. Journal of Applied Statistics, 31(7), 799-815.

Iezzi, G. e. a. (2010). Matemática: Ciência e aplicações, vol. 2, $5^{\circ}$ edn. São Paulo: Atual.

PAIVA, M. R. (2010). Matemática: Paiva, vol. 2. São Paulo: Moderna. 